Pacific Journal of Mathematics

ON CHARACTERISTIC HYPERSURFACES OF SUBMANIFOLDS 


\section{ON CHARACTERISTIC HYPERSURFACES OF SUBMANIFOLDS IN EUCLIDEAN SPACE}

\section{Keti Tenenblat}

The main purpose of this paper is to prove that $M^{n} \subset E^{N}$, where $N=n(n+1) / 2$, the characteristic $(n-1)$-dimensional submanifolds of $M^{n}$ are the asymptotic hypersurfaces.

1. Introduction. The concept of a characteristic submanifold of a given solution for a differential system, was introduced by $\mathrm{E}$. Cartan in his theory of partial differential equations ([2], p. 79). Its importance appears in the treatment of the Cauchy problem.

Given an $n$-dimensional submanifold $M^{n}$ of the Euclidean space $E^{N}$, we can define geometrically the notion of asymptotic submanifolds of $M^{n}$. The asymptotic lines have been used extensively for the study of the geometry of a surface in $E^{3}$. For higher dimension and codimension some results have been obtained, using the generalized concept [3], [4], [9], [10]. It is well known, that the characteristic curves of a surface in $E^{3}$ are the asymptotic lines ([2], p. 143).

In $\S 2$ we start with a brief introduction to the Cartan-Kähler theory of differential equations. Then given a Riemannian manifold $M^{n}$, we consider the differential ideal, whose integral submanifolds determine local isometries of ' $M^{n}$ into $E^{N}, N=n(n+1) / 2$. Next assuming $M^{n} \subset E^{N}$, we characterize the $(n-1)$-dimensional characteristic submanifolds of $M^{n}$.

In $\S 3$, we define the concept of asymptotic submanifolds of $M^{n} \subset E^{N}$, prove the main result and obtain a first order partial differential equation whose solutions are the characteristic hypersurfaces of $M$.

I am grateful to Professor S. S. Chern for helpful conversations.

2. Characteristic submanifold. Let $M$ be an $n$-dimensional differentiable manifold. We denote by $\Lambda_{k}(M)$ the vector space of differential $k$-forms on $M$ and $\Lambda(M)=\sum_{k=0}^{n} \Lambda_{k}(M)$. A differential ideal is an ideal $U$ in $\Lambda(M)$ which is finitely generated, homogeneous (i.e., $U=\sum_{k=0}^{n} U_{k}$ where $U_{k}=U \cap \Lambda_{k}(M)$ ) are closed under exterior differentiation. We assume that $U$ is a differential ideal which does not contain functions i.e., $U_{0}=0$. A $p$-dimensional submanifold $S$ of $M$ is said to be an (p-dimensional) integral submanifold for $U$, if $i^{*}(U)=0$ i.e., $i^{*}\left(U_{p}\right)=0$ where $i: S \rightarrow M$ is the inclusion map.

We denote by $T_{x} M$ the tangent space to $M$ at $x \in M ; G_{x}^{p}(M)$ denotes the Grassman manifold of $p$-dimensional subspaces of $T_{x} M$ 
and $G^{p}(M)=\mathrm{U}_{x \in M} G_{x}^{p}(M)$ is given the usual manifold structure. An element $E_{x}^{p} \in G_{x}^{p}(M)$ is said to be an integral element for $U$, if all the differential forms of $U$ vanish when restricted to the elements of $E_{x}^{p}$.

Let $I_{x}^{p}(U)$ denote the set of $p$-dimensional integral elements for $U$ at $x$, and let $I^{p}(U)=\bigcup_{x \in M} I_{x}^{p}(M)$ be given the topology as a subspace of $G^{p}(M)$. If $E_{x}^{p}$ is an integral element for $U$ generated by $\left\{v_{1}, \cdots, v_{p}\right\}$, we define the polar space $H\left(E_{x}^{p}\right)$ by

$$
H\left(E_{x}^{p}\right)=\left\{v \in T_{x} M ; \phi\left(v, v_{1}, v_{2}, \cdots, v_{p}\right)=0, \forall \phi \in U_{p+1}\right\} .
$$

An integral element $E_{x}^{p}, p \geqq 1$ is said to be ordinary if there exist integral elements $E_{x}^{0}, E_{x}^{1}, \cdots, E_{x}^{p-1}$ with $E_{x}^{0} \subset E_{x}^{1} \subset \cdots E_{x}^{p-1} \subset E_{x}^{p}$ such that $\operatorname{dim} H\left(E_{x}^{i}\right)$ is constant on a neighborhood of $E_{x}^{i}$ in $I^{i}(U)$ for $i=0,1, \cdots, p-1$. A zero-dimensional integral element $E_{x}^{0}$ is said to be regular if $\operatorname{dim} H\left(E_{x}^{0}\right)$ is constant on a neighborhood of $E_{x}^{0}$ in $I^{0}(U)$. A $p$-dimensional integral element $E_{x}^{p}, p \geqq 1$ is said to be regular if it is ordinary and $\operatorname{dim} H\left(E_{x}^{p}\right)$ is constant on a neighborhood of $E_{x}^{p}$ in $I^{p}(U)$. We remark that when $M$ is connected, this definition of regularity is equivalent to Cartan's ([2], pp. 61-67) according to which, an integral element $E_{x}^{p}$ is regular if it is ordinary and $\operatorname{dim} H\left(\begin{array}{l}p \\ x\end{array}\right)$ is equal to the dimension of a generic $p$-dimensional ordinary integral element.

It follows from Cartan-Kähler theorem ([2, pp. 68-74], [7, p. 26]) under the assumption that the manifold $M$ and the differential forms are analytic, that given a $q$-dimensional ordinary integral element $E_{x}^{q}$, then there exists a $q$-dimensional integral submanifold $S$, which contains $x$ ond satisfies the requirement $T_{x} S=E_{x}^{q}$.

An integral submanifold $S$ for $U$ is said to be singular if $\forall x \in S$, the integral element $T_{x} S$ is not ordinary. We remark, that an integral submanifold $S$ may be singular because none of its points is regular, or none of its tangential subspaces of dimension one, or two, $\cdots$, etc., or $p-1$ is regular, where $p$ is the dimension of $S$. Hence one may have different classes of singular integral submanifolds, whose degree of singularity decreases in a certain sense when one goes from one class to the next one.

Let $S$ be a $p$-dimensional nonsingular integral submanifold for $U$, a submanifold $\bar{S} \subset S$ of dimension $q<p$ is called characteristic if $\forall x \in \bar{S}$, the integral element $T_{x} \bar{S}$ is not regular.

The concepts introduced above, can be found with more details in [2] and [7]. The Cartan-Janet theorem [1], [6] asserts that any real analytic, $n$-dimensional, Riemannian manifold can be locally mapped by a real analytic isometric embedding, into a Euclidean space $E^{N}$ of dimension $N=n(n+1) / 2$. In what follows we consider the differential ideal, whose integral submanifolds give local isome- 
tries of $M$ into $E^{N}$. Next assuming $M \subset E^{N}$, we characterize the $(n-1)$-dimensional characteristic submanifolds of $M$. We adopt the following indices convention

$$
\begin{aligned}
& 1 \leqq i, j, k, l \leqq n ; \quad n+1 \leqq \lambda, \mu, \alpha \leqq N ; \\
& 1 \leqq I, J, K \leqq N ; \quad N=n(n+1) / 2
\end{aligned}
$$

and the summation convention with regard to repeated indices.

Let $M$ be an $n$-dimensional Riemannian manifold with metric $g$. Let $F(M)$ denote the bundle of orthonormal frames over $M$, with the usual manifold structure. Under the action of the orthogonal group $O(n), F(M)$ is a principal fiber bundle over $M$, with structural group $O(n)$. Let $\pi: F(M) \rightarrow M$ be the usual projection. We define the canonical forms $\omega^{1}, \cdots, \omega^{n}$ on $F(M)$ by

$\pi_{* z}(v)=\omega^{i}(v) e_{i}$ where $z=\left(x, e_{1}, \cdots, e_{n}\right) \in F(M)$ and $v \in T_{z}(F(M))$, hence $\pi^{*} g=\sum_{i} \omega^{i} \otimes \omega^{i}$. The connection forms $\omega_{i}^{j}$ on $F(M)$ are uniquely defined by

$$
d \omega^{i}=\omega^{j} \wedge \omega_{j}^{i}, \quad \omega_{i}^{j}+\omega_{j}^{i}=0 .
$$

Finally, if we consider

$$
\Omega_{i}^{j}=d \omega_{i}^{j}-\omega_{i}^{k} \wedge \omega_{k}^{j}
$$

then there exist functions $R_{i j_{k l}}$, the components of the Riemann curvature tensor, defined on $F(M)$ such that

$$
\Omega_{i}^{j}=-\frac{1}{2} R_{i j k l} \omega^{k} \wedge \omega^{l}, \quad R_{i j k l}=-R_{i j l k} .
$$

Similarly for $E^{N}$, we denote by $F\left(E^{N}\right)$ the bundle of orthonormal frames over $E^{N}, \bar{\pi}: F\left(E^{N}\right) \rightarrow E^{N}$ the projection, $\bar{\omega}^{I}$ the canonical forms on $F\left(E^{N}\right), \bar{\omega}_{I}^{J}$ the connection forms on $F\left(E^{N}\right)$.

We consider the product manifold $B=F(M) \times F\left(E^{N}\right)$, and define the differential ideal on $B$. Let $\rho: B \rightarrow F(M)$ and $\bar{\rho}: B \rightarrow F\left(E^{N}\right)$ be the usual projections. Using $\rho$ and $\bar{\rho}$ we can pull the differential forms $\omega^{i}, \omega_{i}^{j}, \bar{\omega}^{I}, \bar{\omega}_{I}^{J}$ back to $B$, we will denote the pulled-back forms by the same symbols. Let $U$ be the differential ideal on $B$ generated by

$$
\begin{aligned}
& \bar{\omega}^{i}-\omega^{i} \\
& \bar{\omega}^{\lambda}
\end{aligned}
$$

$$
\begin{aligned}
& \bar{\omega}_{i}^{j}-\omega_{i}^{j} \\
& \omega^{i} \wedge \bar{\omega}_{i}^{\lambda} \\
& \bar{\omega}_{i}^{\lambda} \wedge \bar{\omega}_{\lambda}^{j}+\frac{1}{2} R_{i j l k} \omega^{l} \wedge \omega^{k} .
\end{aligned}
$$


We remark that there is a left action of $O(n)$ on $B$ which preserves the differential ideal $U$. Namely if $A=\left(a_{i j}\right) \in O(n)$ we consider $L_{A}: B \rightarrow B$, which associates to

$$
z=\left(\left(x, e_{1}, \cdots, e_{n}\right),\left(\bar{x}, \bar{e}_{1}, \cdots, \bar{e}_{N}\right)\right) \in B
$$

the point

$$
\begin{aligned}
L_{A}(z)= & \left(\left(x, \sum_{i} a_{1 i} e_{i}, \cdots, \sum_{i} a_{n i} e_{i}\right),\right. \\
& \left.\left(\bar{x}, \sum_{i} a_{l i} \bar{e}_{i}, \cdots, \sum_{i} a_{n i}, \bar{e}_{i}, \bar{e}_{n+1}, \cdots, \bar{e}_{N}\right)\right) .
\end{aligned}
$$

It is not difficult to verify that $L_{A}^{*}\left(U \cap \Lambda_{1}(B)\right) \subset U \cap \Lambda_{1}(B)$ and hence $L_{A}^{*}(U)=U$.

Since we want to determine the $(n-)$-dimentional characteristic submanifolds of $M^{n} \subset E^{N}$, we start characterizing the nonregular $(n-1)$-dimensional integral elements $E_{z}^{n-1}$ for $U$ in $B$, whose projections $\pi_{*} \circ \rho_{*}\left(E_{z}^{n-1}\right)$ are $(n-1)$-dimensional. This characterization is obtained in Lemma 1 (c).

Let $p$ be an integer $0 \leqq p<n$, we adopt the additional index conventions

$$
1 \leqq a, b, c \leqq p ; \quad p+1 \leqq r, s, t \leqq n .
$$

Suppose that $E_{z}^{p}$ is a $p$-dimentional integral element for $U$, generated by vectors $e_{1}, \cdots, e_{p}$ such that

$$
\omega^{a}\left(e_{b}\right)=\delta_{b}^{a}, \quad \omega^{r}\left(e_{b}\right)=0 .
$$

If we denote, $h_{i a}^{\lambda}=\bar{\omega}_{i}^{\lambda}\left(e_{a}\right)$ then it follows, from the fact that the generators of $U$ vanish when restricted to $E_{z}^{p}$, that

$$
\begin{gathered}
h_{a b}^{\lambda}=h_{b a}^{\lambda} \\
\sum_{\lambda}\left(h_{i a}^{\lambda} h_{j b}^{\lambda}-h_{i b}^{\lambda} h_{j a}^{\lambda}\right)-R_{i j a b}=0 .
\end{gathered}
$$

Denote by

$$
H_{i a}=\left(h_{i a}^{n+1}, \cdots, h_{i a}^{N}\right)
$$

the vector in the $(N-n)$-dimentional Euclidean space.

Let $J^{p}$ denote the set of $p$-dimensional integral elements $E_{z}^{p}$, which satisfy the following conditions:

1. $\omega^{1} \wedge \cdots \wedge \omega^{p} \neq 0$ and $\omega^{p+1}=\cdots=\omega^{n}=0$ when restricted to $E_{z}^{p}$.

2. the vectors $\left\{H_{m a}: 1 \leqq a \leqq p, a \leqq m \leqq n-1\right\}$ are linearly independent. Let $V^{p}=\left\{E_{z}^{p} \in I^{p}(U): L_{A^{*}}\left(E_{z}^{p}\right) \in J^{p}\right.$ for some $\left.A \in O(n)\right\}$. 
Then $V^{p}$ is an open subset of $I^{p}(U)$. Part of the next lemma is proved following ([5], with the obvious modifications).

LEMMA 1.

(a) If $0 \leqq p<n$, then $\operatorname{dim} H\left(E_{z}^{p}\right)$ is constant on $V^{p}$;

(b) For $0 \leqq p<n$, if $E_{z}^{p} \in V^{p}$, then it is a regular element;

(c) If $p=n-1$, and $E_{z}^{n-1}$ is an integral element such that $\pi_{*} \circ \rho_{*}\left(E_{z}^{n-1}\right)$ is $(n-1)$-dimensional, then $E_{z}^{n-1}$ is regular if and only if $E_{z}^{n-1} \in V^{n-1}$.

Proof. (a) Since $L_{A}^{*}(U)=U$ it suffices to show that $\operatorname{dim} H\left(E_{z}^{p}\right)$ is constant on $J^{p}$. Assume that $E_{z}^{p}$ is generated by $e_{1}, \cdots, e_{p}$ such that $\omega^{a}\left(e_{b}\right)=\delta_{b}^{a}$ and $\omega^{r}\left(e_{b}\right)=0$. We consider the polar space

$$
\begin{aligned}
H\left(E_{z}^{p}\right) & =\left\{v \in T_{z} B ; \phi\left(v, e_{1}, \cdots, e_{p}\right)=0 \forall \phi \in U_{n}\right\} \\
& =\left\{v \in T_{z} B ; \phi_{1}(v)=0 \text { and } \phi_{2}\left(v, e_{a}\right)=0 \forall \phi_{1} \in U_{1}, \phi_{2} \in U_{2}\right\}
\end{aligned}
$$

where last equality follows from the fact that $U$ is generated by $\left.{ }^{*}\right)$. Hence $H\left(E_{z}^{p}\right)$ consists of vectors $v \in T_{z} B$ which satisfy the following system of equations:

$$
\begin{gathered}
\bar{\omega}^{i}(v)-\omega^{i}(v)=0 \\
\bar{\omega}^{\lambda}(v)=0 \\
\bar{\omega}_{i}^{j}(v)-\omega_{i}^{j}(v)=0 \\
h_{i a}^{\lambda} \omega^{i}(v)-\bar{\omega}_{a}^{\lambda}(v)=0 \\
\sum_{\lambda} h_{j a}^{\lambda} \bar{\omega}_{i}^{\lambda}(v)+\sum_{\lambda} h_{i a}^{\lambda} \bar{\omega}_{\lambda}^{j}(v)-R_{i j l a} \omega^{l}(v)=0, \quad i<j .
\end{gathered}
$$

If we specify $\omega^{i}(v), \omega_{i}^{j}(v)$ then equations (3)-(6) will uniquely determine $\bar{\omega}^{I}(v), \bar{\omega}_{i}^{\jmath}(v)$ and $\bar{\omega}_{a}^{\lambda}(v)$. Moreover we remark that for $1 \leqq i, j \leqq p$, equation (7) is an immediate consequence of (1), (2) and (6). So we need only to consider (7) where $1 \leqq i \leqq p, p+1 \leqq j \leqq n$ and $p+1 \leqq i<j \leqq n$, i.e.,

$$
\begin{aligned}
& \sum_{\lambda} h_{s a}^{\lambda} \bar{\omega}_{b}^{\lambda}(v)+\sum_{\lambda} h_{b a}^{\lambda} \bar{\omega}_{\lambda}^{s}(v)-R_{b s l a} \omega^{l}(v)=0 \\
& \sum_{\lambda} h_{s a}^{\lambda} \bar{\omega}_{t}^{\lambda}(v)+\sum_{\lambda} h_{t a}^{\lambda} \bar{\omega}_{\lambda}^{s}(v)-R_{t s l a} \omega^{l}(v)=0 .
\end{aligned}
$$

Since in (8), for $a \neq b$, interchanging $a$ and $b$ does not modify the equation, we need only to consider

$$
\begin{array}{ll}
\sum_{\lambda} h_{b a}^{\lambda} \bar{\omega}_{s}^{\lambda}(v)=\left(\sum_{\lambda} h_{s a}^{\lambda} h_{i b}^{\lambda}-R_{b s i a}\right) \omega^{i}(v), & a \leqq b \\
\sum_{\lambda} h_{s a}^{\lambda} \bar{\omega}_{t}^{\lambda}(v)-\sum_{\lambda} h_{t a}^{\lambda} \bar{\omega}_{s}^{\lambda}(v)=R_{t s i a} \omega^{i}(v), & s<t .
\end{array}
$$


Denote the vectors

$$
H_{i}(v)=\left(\bar{\omega}_{i}^{n+1}(v), \cdots, \bar{\omega}_{i}^{N}(v)\right) .
$$

We determine the vectors $H_{p+1}(v), \cdots, H_{n}(v)$ so that they satisfy (9) and (10). The system (9) determines the dot product of $H_{p+1}(v)$ with the $p(p+1) / 2$ linearly independent vectors $H_{b a}, a \leqq b$. Once we have chosen a particular $H_{p+1}(v)$ which satisfies this liner system of rank $p(p+1) / 2$, the dot product of $H_{p+2}(v)$ with each of the $p(p+1) / 2+p$ linearly independent vectors $\left\{H_{m a}: 1 \leqq a \leqq p, a \leqq m \leqq p+1\right\}$ is completely determined by (9) and (10). We continue in this fashion. Finally we find that the dot product of $H_{n}(v)$ with each of the $p(p+1) / 2+p(n-p-1)$ linearly independent vectors $\left\{H_{m a}: 1 \leqq a \leqq p\right.$, $a \leqq m \leqq n-1\}$ is completely determined. Hence we find that $\bar{\omega}_{t}^{\lambda}(v)$ must satisfy a consistent system of linear equations which has rank $n p(n-p) / 2$. The polar system of $E_{z}^{p}$ consists of these equations together with (3)-(6). Hence $\operatorname{dim} H\left(E_{z}^{p}\right)$ depends only on $n$ and $p$ whenever $E_{z}^{p} \in J^{p}$.

(b) Suppose that $E_{z}^{p} \in J^{p}$ is generated by $e_{1}, \cdots, e_{p}$, such that $\omega^{a}\left(e_{b}\right)=\delta_{b}^{a}$ and $\omega^{r}\left(e_{b}\right)=0$. If $0 \leqq q \leqq p$, we let $E_{z}^{q}$ be the $q$-dimensional integral element generated by $e_{1}, \cdots, e_{q}$. Then $E_{z}^{q} \in J^{q}$ and hence $\operatorname{dim} H\left(E_{z}^{q}\right)$ is constant in a neighborhood of $E_{z}^{q}$ in $I^{q}(U)$. It follows that $E_{z}^{p}$ is regular. Consequently if $E_{z}^{p} \in V^{p}$, then it is a regular integral element.

(c) From (b) we only need to prove that if $E_{z}^{n-1}$ is a regular integral element then $E_{z}^{n-1} \in V^{n-1}$. Since $\pi_{*} \circ \rho_{*}\left(E_{z}^{n-1}\right)$ is $(n-1)$ dimensional, we can find an element $A \in O(n)$ such that $\omega^{n}=0$ on $L_{A^{*}}\left(E_{z}^{n-1}\right)$. Hence, we can assume that $E_{z}^{n-1}$ is generated by $e_{1}, \cdots, e_{n-1}$, such that $\omega^{a}\left(e_{b}\right)=\delta_{b}^{a}$ and $\omega^{n}\left(e_{b}\right)=0$, where $1 \leqq a, b \leqq n-1$. Since $E_{z}^{n-1}$ is regular, it follows that $\operatorname{dim} H\left(E_{z}^{n-1}\right)$ is constant in a neighborhood of $E_{z}^{n-1}$ in $I^{n-1}(U)$. The polar system of $E_{z}^{n-1}$ is given by (3)-(6) and (7) reduces to

$$
\sum_{\lambda} h_{b a}^{\lambda} \bar{\omega}_{n}^{\lambda}(v)=\left(\sum_{\lambda} h_{n a}^{\lambda} h_{i b}^{\lambda}-R_{b n i a}\right) \omega^{i}(v), \quad a \leqq b .
$$

As in (a) if we specify $\omega^{i}(v), \omega_{i}^{j}(v)$ then $\bar{\omega}^{I}(v), \bar{\omega}_{i}^{j}(v)$ and $\bar{\omega}_{a}^{\lambda}(v)$ will be uniquely determined by (3)-(6). Moreover the $n(n-1) / 2$ components $\bar{\omega}_{n}^{\lambda}(v)$ must satisfy the linear system (11) which has exactly $n(n-1) / 2$ equations. Hence, if $\operatorname{dim} H\left(E_{z}^{n-1}\right)$ is constant in a neighborhood of $E_{z}^{n-1}$, then the determinant of the coefficient matrix in (11) is nonzero, i.e., the vectors $\left\{H_{b a}: 1 \leqq a \leqq b \leqq n-1\right\}$ are linearly independent, which implies $E_{z}^{n-1} \in J^{n-1}$.

Let $M$ be an $n$-dimensional Riemannian manifold and $f: M \rightarrow E^{N}$ an isometric imbedding. If $x_{0} \in M$, there exists a neighborhood $V$ of 
$x_{0}$ in $M$ and a section $\bar{\sigma}: V \rightarrow F\left(E^{N}\right)$ such that if $\bar{\sigma}(x)=\left(f(x), \bar{e}_{1}(x)\right.$, $\left.\cdots, \bar{e}_{N}(x)\right)$, then $\bar{e}_{1}(x), \cdots, \bar{e}_{n}(x)$ are tangent to $f(M)$. We consider the section $\sigma: V \rightarrow F(M)$, defined by $\sigma(x)=\left(x, e_{1}(x), \cdots, e_{n}(x)\right)$ where $f_{*}\left(e_{i}(x)\right)=\bar{e}_{i}(x)$. For simplicity, we denote by $\omega^{i}, \omega_{i}^{j}$ the differential forms $\sigma^{*} \omega^{i}, \sigma^{*} \omega_{i}^{j}$ induced on $V$ and similarly $\bar{\omega}^{I}, \bar{\omega}_{I}^{J}$ will denote the pulled-back forms $\bar{\sigma}^{*} \bar{\omega}^{I}, \bar{\sigma}^{*} \bar{\omega}_{I}^{J}$ on $V$. Consider the map $\Gamma: V \rightarrow B$ defined by $\Gamma(x)=(\sigma(x), \bar{\sigma}(x))$. Since $f$ is an isometry, $\Gamma(V)$ is an integral submanifold for $U$ in $B$. We say that a $q$-dimensional vector space $L^{q} \subset T_{x_{0}} M, 0 \leqq q<n$ is regular if $\Gamma_{*}(L)$ is a regular integral element for $U$. Similarly, a $q$-dimensional submanifold $S$ of $V$ is said to be characteristic, if $\Gamma(S)$ is a characteristic submanifold of $\Gamma(V)$. The characteristic hypersurfaces of $M$ have at each point a nonregular tangent space. Our next lemma characterizes the nonregular $(n-1)$-dimensional spaces tangent to $M$.

We denote the matrix $H^{\lambda}=\left(h_{i j}^{\lambda}\right)$ where $h_{i j}^{\lambda}=\bar{\omega}_{i}^{\lambda}\left(e_{j}\right)$. Moreover, given a matrix $A$, we denote by $A_{b}$ the $b$ th row of $A$ and $A_{b}^{t}$ denotes the transpose of $A_{b}$. Assume $\Gamma(V)$ is not a singular integral submanifold for $U$, then as an immediate concequence of Lemma 1(c), we obtain

LeMma 2. Let $u_{i} \omega^{i}=0$ be an $(n-1)$-dimensional subspace of $T_{x_{0}} M$. We may assume that $\sum_{i=1}^{n} u_{i}^{2}=1$. Choose $A=\left(a_{i j}\right) \in O(n)$ such that $a_{n i}=u_{i}$. Then $u_{i} \omega^{i}=0$ is nonregular if and only if the vectors

$$
\left(A_{a} H^{n+1} A_{b}^{t}, \cdots, A_{a} H^{N} A_{b}^{t}\right), \quad 1 \leqq a \leqq b \leqq n-1
$$

are linearly dependent, as vectors in $E^{N-n}$.

We remark that this condition determines a first order partial differential equation, and the characteristic hypersurfaces of $M$ are the solutions of this equation. In the next section as a consequence of Lemma 3, the partial differential equation will be given in another form, which will not involve the choice of matrix $A$.

3. Asymptotic submanifolds; proof of main result. Let $M$ be an $n$-dimensional $C^{\infty}$ submanifold of $E^{N}, N=n(n+1) / 2$ with the induced metric and such that the inclusion $i: M \rightarrow E^{N}$ is nondegenerate. Let $x \in M$ and denote by $s$ the second fundamental form. A $q$ dimensional $0<q<n$ linear subspace $L$ of the tangent space $T_{x} M$ is called asymptotic if there exists a vector $\xi$ normal to $T_{x} M$ such that $\langle s(X, Y), \xi\rangle=0, \forall X, Y \in L$ where $\langle$,$\rangle denotes the Euclidean$ metric. If $L$ is of codimension one, we have an asymptotic hyperplane at $x$. A $q$-dimensional submaniford $V$ of $M, q<n$ is called asymptotic at $x \in V$ if $T_{x} V$ is asymptotic and asymptotic if this is 
true for each $x \in V$. It is not difficult to see that $V$ is an asymptotic hypersurface of $M$ if and only if there exists a normal to the osculating space of $V$, which is also normal $M$. The notation of asymptotic submanifold in a more general context can be found in [4].

Let $e_{1}, \cdots, e_{N}$ be an orthonormal frame defined on a neighborhood of $x \in M$, such that $e_{1}, \cdots, e_{n}$ are tangent to $M$ and $e_{n+1}, \cdots, e_{N}$ are normal to $M$. Let $\omega^{1}, \cdots, \omega^{N}$ be the dual frame. With the same indices convention as in $\S 2$, we denote by $h_{i j}^{\lambda}=\omega_{i}^{\lambda}\left(e_{j}\right)$ where $\omega_{i}^{\lambda}$ are the connection forms. It follows from the definition that a hyperplane $u_{i} \omega^{i}=0$ is asymptotic if and only if the second fundamental forms $h_{i j}^{i} \omega^{i} \otimes \omega^{j}$ are linearly dependent when restricted to $u_{i} \omega^{i}=0$.

The following algebraic lemma shows that the condition obtained in Lemma 2 is eqivalent to saying that $u_{i} \omega^{i}=0$ is asymptotic. As in $\S 2$ given a matrix $A$ we denote by $A_{b}$ the bth row of $A$ and $A_{b}^{t}$ denotes the transpose of $A_{b}$.

LeMma 3. Let $\phi^{\lambda}$ be $n \times n$ symmetric matrices $\lambda=n+1, \cdots, N$. $N=n(n+1) / 2$ and let $A=\left(a_{i j}\right) \in O(n)$. Then the vectors

$$
\left(A_{b} \varphi^{n+1} A_{c}^{t}, \cdots, A_{b} \varphi^{N} A_{c}^{t}\right), \quad 1 \leqq b \leqq c \leqq n-1
$$

are linearly dependent, as vectors in $E^{N-n}$, if and only if the quadratic forms $\varphi_{i j} \omega^{i} \otimes \omega^{j}$ are linearly dependent when restricted to $a_{n i} \omega^{i}=0$, where $\omega^{i}$ are $n$ independent 1-forms.

Proof. The vectors $\left(A_{b} \varphi^{n+1} A_{c}^{t}, \cdots, A_{b} \Phi^{N} A_{c}^{t}\right)$ are linearly dependent iff $\exists \alpha_{\lambda} \in \boldsymbol{R}$ not all zero, such that

$$
A_{b}\left(\sum_{\lambda=n+1}^{N} \alpha_{\lambda} \varphi^{\lambda}\right) A_{c}^{t}=0, \quad \forall 1 \leqq b \leqq c \leqq n-1 .
$$

We denote by $D$ the matrix $D=\sum_{\lambda} \alpha_{\lambda} \varphi^{\lambda}$ and $W=\left(\omega^{1}, \cdots, \omega^{n}\right)$. We will prove that $A_{b} D A_{c}^{t}=0 \quad \forall 1 \leqq b \leqq c \leqq n-1$ if and only if $W D W^{t}=0$ whenever $A_{n} W^{t}=0$.

Consider

$$
W D W^{t}=W A^{t}\left(A D A^{t}\right) A W^{t} .
$$

Suppose $A_{b} D A_{c}^{t}=0, \forall 1 \leqq b \leqq c \leqq n-1$, then since $D$ is symmetric

$$
W D W^{t}=\left[W A_{1}, \cdots, W A_{n-1}^{t} W A_{n}^{t}\right]\left[\begin{array}{cc}
A_{1} D A_{n}^{t} \\
\vdots \\
A_{n-1} D A_{n}^{t} \\
A_{n} D A_{1}^{t} \cdots & A_{n} D A_{n}^{t}
\end{array}\right]\left[\begin{array}{c}
A_{1} W^{t} \\
\vdots \\
A_{n-1} W^{t} \\
A_{n} W^{t}
\end{array}\right] .
$$


Hence if $A_{n} W^{t}=0$ then $W D W^{t}=0$, i.e., the quadratic forms $W \phi^{\lambda} W^{t}$ are linearly dependent whenever $A_{n} W^{t}=0$.

Conversely, suppose $W D W^{t}=0$ when $A_{n} W^{t}=0$, then it follows from (12) that

$$
0=\sum_{b=1}^{n-1} A_{b} D A_{b}^{t}\left(\sum_{k=1}^{n} a_{b k} \omega^{k}\right)^{2}+2 \sum_{\substack{b, c=1 \\ b<c}}^{n-1} A_{b} D A_{c}^{t}\left(\sum_{k, l=1}^{n} a_{b k} a_{c l} \omega^{k} \otimes \omega^{l}\right) .
$$

Let $e_{i}$ be the dual basis of $\omega^{i}$, i.e., $\omega^{i}\left(e_{j}\right)=\delta_{j}^{i}$. If we evaluate (13) at the pair $\left(e_{k}, e_{k}\right)$ we get

$$
\sum_{b=1}^{n-1} A_{b} D A_{b}^{t} a_{b k}^{2}+2 \sum_{\substack{b, c=1 \\ b<c}}^{n-1} A_{b} D A_{c}^{t} a_{b k} a_{c k}=0, \quad \forall k=1, \cdots, n .
$$

Adding over $k$, since $A \in O(n)$ we get

$$
\sum_{b=1}^{n-1} A_{b} D A_{b}^{t}=0 \text {. }
$$

If we apply (13) to the pairs $\left(e_{k}, e_{l}\right)\left(e_{l}, e_{k}\right) l \neq k$ and subtract we get

$$
\sum_{\substack{b, c=1 \\ b<c}}^{n-1} A_{b} D A_{c}^{t}\left(a_{b k} a_{c l}-a_{b l} a_{c k}\right)=0, \quad \forall 1 \leqq k \leqq l \leqq n .
$$

This is an homogeneous linear system of $n(n-1) / 2$ equations with $(n-1)(n-2) / 2$ unknowns $A_{b} D A_{c}^{t}, 1 \leqq b<c \leqq n-1$. We claim that the rank of this system is $(n-1)(n-2) / 2$. In fact, otherwise it follows from Sylvester-Franke theorem on determinants ([8], p. 94, take $m=2$ ), that the cofactor of $a_{n i}$ in $A$ is zero, $\forall i=1, \cdots, n$, which contradicts the fact that $\operatorname{det} A \neq 0$. Hence from (15) we have that

$$
A_{b} D A_{c}^{t}=0, \quad 1 \leqq b<c \leqq n-1 .
$$

Now (13) reduces to

$$
\sum_{b=1}^{n-1} A_{b} D A_{b}^{t}\left(\sum_{k=1}^{n} a_{b k} \omega^{k}\right)^{2}=0
$$

and from (14) we have

$$
A_{n-1} D A_{n-1}^{t}=-\sum_{b=1}^{n-2} A_{b} D A_{b}^{t} .
$$

If we substitute (18) in (17) we get

$$
\sum_{b=1}^{n-2} A_{b} D A_{b}^{t}\left(\sum_{k=1}^{n}\left(a_{b k}-a_{n-1 k}\right) \omega^{k}\right)\left(\sum_{k=1}^{n}\left(a_{b k}+a_{n-1 k}\right) \omega^{k}\right)=0 .
$$

Applying this equation to the pairs of vectors $\left(e_{k}, e_{l}\right),\left(e_{l}, e_{k}\right), l \neq k$ and subtracting we get 


$$
\sum_{b=1}^{n-2} A_{b} D A_{b}^{t}\left(a_{b k} a_{n-1 l}-a_{n-1 k} a_{b l}\right)=0, \quad 1 \leqq k<l \leqq n .
$$

This is a linear system of $n(n-1) / 2$ equations with $n-2$ unknowns $A_{b} D A_{b}^{t}, 1 \leqq b \leqq n-2$. The rank of this system is $n-2$. Otherwise, using Laplace's development of a determinant in the general version (i.e., the determinant is a linear function of the minors comprised in any number of lines) we get that the system (15) has rank lower than $(n-1)(n-2) / 2$, which is a contradiction. Therefore $A_{b} D A_{b}^{t}=0$ for $b=1, \cdots, n-2$ and finally from (16) and (18) we conclude that $A_{b} D A_{t}^{c}=0 \quad \forall 1 \leqq b \leqq c \leqq n-1$.

Let $f: M \rightarrow E^{N}$ be an isometric embedding, with the same notation as in 2, we say that $f$ is singular if $\forall x \in M, \Gamma_{*}\left(T_{x} M\right)$ is not an ordinary integral element for $U$ in $B$. Then our main result follows immediately from Lemmas 2 and 3:

THEOREM. Let $f: M \rightarrow E^{N}$ be a nonsingular isometric imbedding. An $(n-1)$-dimensional submanifold of $M$ is characteristic if and only if it is asymptotic.

We remark that $f$ being nonsingular implies that $f$ is nondegenerate, but for $n>2$ it may exist a nondegenerate isometric imbedding which is singular; in this case all hypersurfaces would be asymptotic.

We observe that it is not difficult to prove that $u_{i} \omega^{i}=0$ is asymptotic if and only if there exist real numbers $a_{\lambda}, b_{i}$ not all zero, such that

$$
a_{\lambda} h_{i j}^{\lambda} \omega^{i} \otimes \omega^{j} \equiv u_{i} \omega^{i} \otimes b_{j} \omega^{j} .
$$

This reduces to a homogeneous equation in $u_{i}$ of degree $n, P\left(u_{1}, u_{2}\right.$, $\left.\cdots, u_{n}\right)=0$. In order to describe the polynomial $P$ we consider the matrices

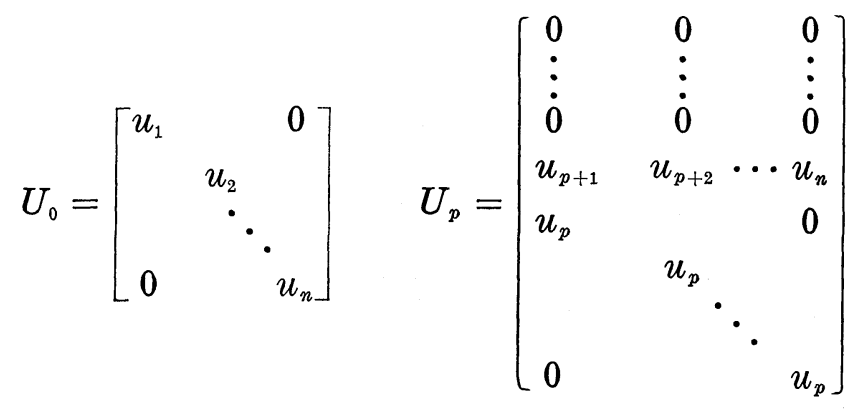

where $U_{p}$ has the first $(p-1)$ rows equal to zero, $1 \leqq p \leqq n-1$ 


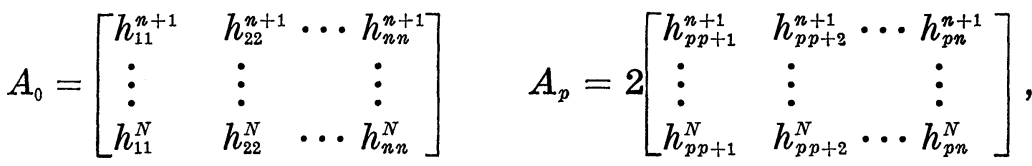

$$
\begin{aligned}
& 1 \leqq p \leqq n-1 \text {. }
\end{aligned}
$$

Then

$$
P\left(u_{1}, u_{2}, \cdots, u_{n}\right)=\operatorname{det}\left[\begin{array}{llll}
U_{0} & U_{1} & \cdots & U_{n-1} \\
A_{0} & A_{1} & \cdots & A_{n-1}
\end{array}\right]=0 \text {. }
$$

Hence the characteristic hypersurfaces of $M$ are the solutions of the first order partial differential equation defined by $P\left(u_{1}, \cdots, u_{n}\right)=0$. For $n=3$ this equation was obtained by Cartan ([2], p. 208).

\section{REFERENCES}

1. É Cartan, Sur la possibilité de plonger un espace riemannien donné dans un espace euclidien, Ann. Soc. Polon. Math., 6 (1927), 1-7.

2. - Les systèmes différentiels extérieurs et leurs applications géométriques, Hermann, Paris, 1945.

3. S. S. Chern and N. Kuiper, Some theorems on the isometric imbeddings of compact Riemann manifolds in Euclidean space, Ann. of Math., 56 (1952), 422-430.

4. H. Jacobowitz, Deformations leaving a hypersurface fixed., Proc. Sympos. Pure Math. Amer. Math. Soc., 23 (1971), 343-351.

5. H. Jacobowitz and J. D. Moore, The Cartan-Janet theorem for conformal embeddings, Indiana Univ. Math. J., 23 (1973), 187-203.

6. M. Janet, Sur la possibilité de plonger un espace riemannien donné dans un espace euclidien, Ann. Soc. Polon. Math., 5 (1926), 38-43.

7. E. Kähler, Einführung in die Theorie der Systeme von Differentialgleichungen, Chelsea, New York, 1949.

8. G. Kowalewski, Einführung in die Determinantentheorie, Chelsea, 1948.

9. J. D. Moore, Isometric immersions of space forms in space forms, Pacific J. Math., 40 (1972), 157-166.

10. K. Tenenblat, A rigidity theorem for three dimensional submanifolds in Euclidean six space, J. Differential Geometry, to appear.

Received June 1, 1977. Partially supported by CNPq.

University of CALIFornia, Berkeley, CA94720

AND

Universidade De Brasilia, 70000 Brasilia, D. F. Brazil 



\section{PACIFIC JOURNAL OF MATHEMATICS}

\section{EDITORS}

RICHARD ARENS (Managing Editor)

University of California

Los Angeles, CA 90024

Charles W. Curtis

University of Oregon

Eugene, OR 97403

C. C. Moore

University of California

Berkeley, CA 94720

\section{J. DugundJI}

Department of Mathematics

University of Southern California

Los Angeles, CA 90007

R. FInN and J. Milgram

Stanford University

Stanford, CA 94305

\section{ASSOCIATE EDITORS}
E. F. BECKENBACH
B. H. NeumanN
F. WOLF
K. YoSHIDA

\section{SUPPORTING INSTITUTIONS}

\author{
UNIVERSITY OF BRITISH COLUMBIA \\ CALIFORNIA INSTITUTE OF TECHNOLOGY \\ UNIVERSITY OF CALIFORNIA \\ MONTANA STATE UNIVERSITY \\ UNIVERSITY OF NEVADA, RENO \\ NEW MEXICO STATE UNIVERSITY \\ OREGON STATE UNIVERSITY \\ UNIVERSITY OF OREGON
}

\author{
UNIVERSITY OF SOUTHERN CALIFORNIA \\ STANFORD UNIVERSITY \\ UNIVERSITY OF HAWAII \\ UNIVERSITY OF TOKYO \\ UNIVERSITY OF UTAH \\ WASHINGTON STATE UNIVERSITY \\ UNIVERSITY OF WASHINGTON
}

The Supporting Institutions listed above contribute to the cost of publication of this Journal, but they are not owners or publishers and have no responsibility for its content or policies.

Mathematical papers intended for publication in the Pacific Journal of Mathematics should be in typed form or offset-reproduced, (not dittoed), double spaced with large margins. Please do not use built up fractions in the text of the manuscript. However, you may use them in the displayed equations. Underline Greek letters in red, German in green, and script in blue. The first paragraph or two must be capable of being used separately as a synopsis of the entire paper. Items of the bibliography should not be cited there unless absolutely necessary, in which case they must be identified by author and journal, rather than by item number. Manuscripts, in triplicate, may be sent to any one of the editors. Please classify according to the scheme of Math. Reviews, Index to Vol. 39. All other communications should be addressed to the managing editor, or Elaine Barth, University of California, Los Angeles, California, 90024.

50 reprints to each author are provided free for each article, only if page charges have been substantially paid. Additional copies may be obtained at cost in multiples of 50 .

The Pacific Journal of Mathematics is issued monthly as of January 1966. Regular subscription rate: $\$ 72.00$ a year (6 Vols., 12 issues). Special rate: $\$ 36.00$ a year to individual members of supporting institutions.

Subscriptions, orders for numbers issued in the last three calendar years, and changes of address should be sent to Pacific Journal of Mathematics, 103 Highland Boulevard, Berkeley, California, 94708. Older back numbers obtainable from Kraus Periodicals Co., Route 100, Millwood, NY 10546.

PUBLISHED BY PACIFIC JOURNAL OF MATHEMATICS, A NON-PROFIT CORPORATION

Printed at Kokusai Bunken Insatsusha (International Academic Printing Co., Ltd.). 8-8, 3-chome, Takadanobaba, Shinjuku-ku, Tokyo 160, Japan.

Copyright (C) 1978 by Pacific Journal of Mathematics

Manufactured and first issued in Japan 


\section{Pacific Journal of Mathematics

Vol. 74, No. $2 \quad$ June, 1978

Aharon Atzmon, Spectral synthesis in some spaces of bounded continuous

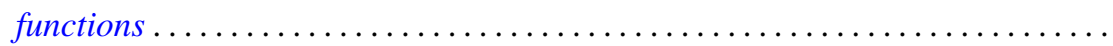

Karl Egil Aubert and Isidor Fleischer, Tensor products of ideal systems and their modules.............................................

Richard F. Basener, Several dimensional properties of the spectrum of a uniform

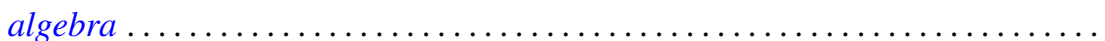

R. H. Bing and Michael Peter Starbird, Super triangulations ............. 307

Andrew Carson, Coherent polynomial rings over regular rings of finite index .....

Robert M. DeVos and Frederick W. Hartmann, Sequences of bounded summability domains .................................................

George Grätzer and R. Padmanabhan, Symmetric difference in abelian groups ....

Robert L. Griess, Jr., A remark about groups of characteristic 2-type and

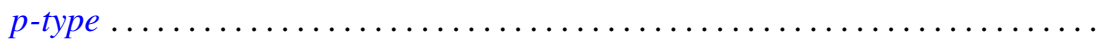

Emil Grosswald and F. J. Schnitzer, A class of modified $\zeta$ and L-functions........

Jutta Hausen and Johnny Albert Johnson, Ideals and radicals of some

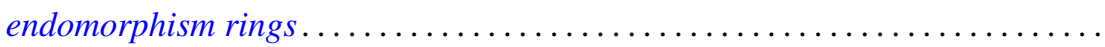

Jean Ann Larson, A solution for scattered order types of a problem of Hagendorf ............................................

Peter A. McCoy, Extremal properties of real biaxially symmetric potentials in $E^{2(\alpha+\beta+2)}$

Héctor Alfredo Merklen, Hereditary crossed product orders .

Hal G. Moore and Adil Mohamed Yaqub, Equational definability of addition in certain rings...

Robert Laurens Moore, Reductivity in $C^{*}$-algebras and essentially reductive operators. . .

Joseph Alvin Neisendorfer, Lie algebras, coalgebras and rational homotopy theory for nilpotent spaces...

William Raymond Nico, Bounded monoids

Richard Paul Osborne, Simplifying spines of 3-manifolds ...

Richard Paul Osborne, The simplest closed 3-manifolds. With an appendix by Osborne and J. Yelle.

Clayton Collier Sherman, The $K$-theory of an equicharacteristic discrete valuation ring injects into the $K$-theory of its field of quotients.... .

Mitchell Herbert Taibleson, The failure of even conjugate characterizations of $H^{1}$

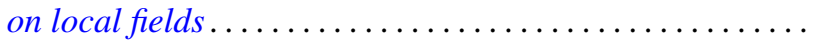

Keti Tenenblat, On characteristic hypersurfaces of submanifolds in Euclidean space ...................................

Jeffrey L. Tollefson, Involutions of Seifert fiber spaces..........

Joel Larry Weiner, An inequality involving the length, curvature, and torsions of a curve in Euclidean $n$-space .......................

Neyamat Zaheer, On generalized polars of the product of abstract homogeneous polynomials.... 